\title{
Revisiting the Functional Specialization of Left Inferior Frontal Gyrus in Phonological and Semantic Fluency: The Crucial Role of Task Demands and Individual Ability
}

\author{
Michael Katzev, ${ }^{1,2}$ Oliver Tüscher, ${ }^{1,2,3}$ Jürgen Hennig, ${ }^{2,4,5}$ Cornelius Weiller, ${ }^{1,2,5}$ and Christoph P. Kaller ${ }^{1,2,5}$ \\ ${ }^{1}$ Department of Neurology, University Medical Center Freiburg, 79106 Freiburg, Germany, ${ }^{2}$ Freiburg Brain Imaging Center, University of Freiburg, 79085 \\ Freiburg, Germany, ${ }^{3}$ Department of Psychiatry, University Medical Center Mainz, 55131 Mainz, Germany, ${ }^{4}$ Medical Physics, Department of Radiology, \\ University Medical Center Freiburg, 79106 Freiburg, Germany, and ${ }^{5}$ BrainLinks-BrainTools Cluster of Excellence, University of Freiburg, 79085 Freiburg, \\ Germany
}

Despite a large body of research, extant findings on the functional role of left inferior frontal gyrus (LIFG) in phonological and semantic fluency are still controversial. Based on cross-study comparisons, a recent meta-analysis of neuroimaging results suggests that posteriordorsal (Brodmann area, BA, 44) and anterior-ventral parts (BA 45) of LIFG contribute differentially to processes of phonologically and semantically cued word retrieval, respectively. In contrast, a subsequent functional magnetic resonance imaging experiment failed to validate the proposed dissociation using a within-subjects design. In particular, no evidence for a specific role of BA 45 in semantic fluency was found. Here, we resolve this apparent controversy by showing that the conflicting findings can be accounted for when considering the influence of task demands and individual ability on resulting functional magnetic resonance imaging activation patterns. By comparing phonological versus semantic fluency, higher activation was robustly observed in BA 44 . For the opposite comparison, higher activation was found in dorsal BA 45; however, this was more pronounced in posterior-dorsal parts of BA 45 for low-performing subjects and was only apparent in anterior-dorsal parts of BA 45 under high demands on controlled semantic retrieval. Our results thus disclose important determinants for detecting a functional segregation of LIFG in verbal fluency that also have implications for the controversial findings in previous lesion studies. Moreover, the present parcellation of dorsal BA 45 corresponds well with anatomical evidence suggesting a subdivision into an anterior (45A) and posterior part (45B) and may therefore represent evidence for its functional significance in humans.

\section{Introduction}

Verbal fluency constitutes a classic neuropsychological test of frontal lobe damage (Milner, 1964; Benton, 1968) that is among the most frequently applied clinical measures in a multitude of brain pathologies (Strauss et al., 2006). It is prototypically assessed in terms of deliberate word generation following either a phonological cue (e.g., letters; F, A, S, etc.) or a semantic cue (e.g., categories; animals, fruits, etc.). However, despite a wealth of research, there is still considerable debate about the frontal lobes' functional role in semantic versus phonological fluency

Received July 3, 2012; revised March 22, 2013; accepted March 27, 2013.

Author contributions: M.K., O.T., C.W., and C.P.K. designed research; M.K., J.H., and C.P.K. performed research; C.P.K. contributed unpublished reagents/analytic tools; M.K. and C.P.K. analyzed data; M.K., C.W., and C.P.K. wrote the paper.

This work was supported by the European Union FP 7 research program (PLASTICISE: Collaborative Project 223524) and the BrainLinks-BrainTools Cluster of Excellence funded by the German Research Foundation (DFG; Grant \#EXC 1086). We thank Anna Eras, Tobias Etzold, Lisa Ewald, Astrid Gössel, Sabrina Huber, Isabella Sing, and Tobias Uhl for assistance during data acquisition and subsequent analysis of verbal responses and Benjamin Rahm and Lena Köstering for valuable discussion of a previous version of the manuscript.

The authors declare no competing financial interests.

Correspondence should be addressed to either Michael Katzev or Christoph Kaller, Department of Neurology, University Medical Center, University of Freiburg, Germany, Breisacher Strasse 64, 79106 Freiburg, Germany, E-mail: michael.katzev@uniklinik-freiburg.de or christoph.kaller@uniklinik-freiburg.de.

DOI:10.1523/JNEUROSCI.3147-12.2013

Copyright $\odot 2013$ the authors $\quad 0270-6474 / 13 / 337837-09 \$ 15.00 / 0$
(Mummery et al., 1996; Paulesu et al., 1997; Troyer et al., 1998; Gourovitch et al., 2000; Schwartz et al., 2003; Baldo et al., 2006; Birn et al., 2010).

Ongoing discussions in the neuropsychological literature focus on the different specificity and sensitivity of the task's two variants for frontal lobe damage (Troyer et al., 1998; Henry and Crawford, 2004; Robinson et al., 2012), whereas a related key issue in the neuroimaging literature concerns whether the differentially involved cognitive processes have dissociable localizations within prefrontal cortex (Mummery et al., 1996; Costafreda et al., 2006; Heim et al., 2008). Based on a systematic review and meta-analysis, Costafreda et al. (2006) suggested that phonological fluency is represented more posterior-dorsally and semantic fluency more anterior-ventrally in left inferior frontal gyrus (LIFG), corresponding approximately to Brodmann areas (BA) 44 and 45 (Costafreda et al., 2006). Testing the proposed dissociation directly in a within-subjects experiment, Heim et al. (2008) did not find supporting evidence. Compared with rest, both conditions elicited overlapping activations in BA 44 and BA 45. However, phonological fluency was found to activate BA 44 stronger than semantic fluency, whereas the inverse contrast did not reveal significant activation differences in any part of LIFG (Heim et al., 2008). 
Here, we propose that these seemingly opposing results of Costafreda et al. (2006) and Heim et al. (2008) may be integrated when the role of task demands and individual ability is considered. Although both fluency tasks share several core processes (e.g., self-monitoring of verbal output or suppression of previously retrieved responses; Azuma, 2004), phonological and semantic fluency differ considerably with respect to the kind of search processes required for successful retrieval (Unsworth et al., 2011). Phonological fluency most likely involves a serial search based on systematic syllabification of initial letters (Mummery et al., 1996; Rende et al., 2002), whereas semantic search is driven by association chains and spreading activations within cue-related subcategories (Gruenewald and Lockhead, 1980). In addition to generating task-relevant subcategories (Rosen and Engle, 1997) and actively shifting between them (Troyer et al., 1997; Reverberi et al., 2006), semantic fluency may thus require additional control processes such as selection of appropriate items from competing alternatives (Thompson-Schill et al., 1997, 1998). Because controlled compared with overlearned semantic retrieval results in selective activation of BA 45 (Amunts et al., 2004), we tested the hypothesis that semantic versus phonological fluency leads to differential activation of BA 45 only under high demands on controlled semantic search and item selection. In addition, the impact of individual ability on LIFG activation was explored (compare Borkowski et al., 1967, Rosen and Engle, 1997).

\section{Materials and Methods}

Subjects. Sixty-two healthy volunteers 18 to 45 years of age (mean, 26.1 years; SD, 6.6 years; 28 females) participated in the present experiment with functional magnetic resonance imaging (fMRI). All subjects were right-handed native German speakers and had normal or corrected-tonormal vision. None of them was under medical treatment or reported a history of neurological or psychiatric disorders. Written informed consent was obtained from all subjects before participation. The study protocol was approved by the local ethics authorities. Subjects received a compensation of 20 Euros for participation in the present experiment, which was part of a larger study on prefrontal functions in healthy and pathological conditions.

Experimental stimuli. The primary objective of this study was to test for a presumed interaction effect between cue type (phonological vs semantic) and task demand (high vs low). To this end, we conducted a behavioral pilot study to select a set of appropriate letter and category cues for the intended experimental manipulation of task difficulty. For the phonological condition in the pilot experiment, preselection of 16 letter cues was informed by the Mannheim Corpus of the German language provided by the CELEX database (Max Planck Institute for Psycholinguistics, Nijmegen, The Netherlands; http://celex.mpi.nl). The Mannheim Corpus comprises frequency estimations for a large set of existing words in spoken German, and thus allows for a first indication of the expected difficulty of individual letter cues. Before letter selection from the alphabet, the consonant $\mathrm{J}$ and all vowels $(\mathrm{A}, \mathrm{E}, \mathrm{I}, \mathrm{U}, \mathrm{O})$ were excluded. Thereafter, consonants $\mathrm{Y}, \mathrm{X}, \mathrm{Q}$, and $\mathrm{C}$ were excluded given their relatively infrequent occurrence as initial letters of German nouns (maximum of 332 counts per one million nouns, exclusive of compound words). Frequencies of the remaining 16 consonants $(\mathrm{N}, \mathrm{L}, \mathrm{Z}, \mathrm{D}, \mathrm{R}, \mathrm{T}, \mathrm{W}, \mathrm{V}, \mathrm{K}, \mathrm{P}, \mathrm{H}$, G, F, M, B, S) were distributed from 1112 to 8595 counts, thus providing a sufficient range of difficulty for an empirical selection of letter cues with high and low task demands. For the semantic condition in the pilot experiment, preselection of 16 category cues was based on normative data for 40 semantic fluency categories as assessed in a sample of German students by Mannhaupt (1983). Among other indices, Mannhaupt (1983) provided estimations of average production norms for individual categories within a time interval of $40 \mathrm{~s}$. Preselection of semantic cues included 8 categories with a presumably high task demand (average retrieval of $\sim 8-10$ category members: liquids, flowers, tools, kitchen utensils, toys, accommodation, furniture, trees) and 8 categories with a presumably low task demand (average retrieval of $\sim 12-14$ category members: hobbies, vegetables, professions, foods, vehicles/means of transport, musical instruments, quadrupeds, relatives).

During the pilot experiment, subjects were asked to overtly generate as many nouns/category members per cue as possible within a time limit of $1 \mathrm{~min}$. Verbal and written instructions emphasized that using proper names, morphological variations, or repetitions of words previously retrieved in the same trial was not allowed (Lezak et al., 2004; Strauss et al., 2006). Letter and category trials were blocked, but individual cues were presented separately with short breaks in between trials. Half of the subjects started with the letter cues followed by the category cues, whereas the other half was presented with the reversed order. Within cue-type blocks, position and order of presentation of individual letter/category cues was counterbalanced across subjects. Verbal responses were digitally recorded for subsequent analysis using custom-built software.

Selection of cue stimuli for the fMRI study was based on the behavioral results of the pilot experiment. For the intended $2 \times 2$ factorial manipulation of cue type (phonological/letters vs semantic/categories) and task demand (high vs low), four cues with almost comparable levels of empirical difficulty were chosen for each of the four resulting cells of the experimental design. Due to global differences in task difficulty between phonological and semantic fluency, manipulations of task demand for the two conditions cannot be matched directly; that is, phonological compared with semantic fluency conditions are generally harder to accomplish (Mitrushina et al., 1999; Lezak et al., 2004). To account for these global differences, we applied a selection strategy that provided a comparable level of difficulty for low-demanding phonological and high-demanding semantic cues and a comparable slope of difficulty levels for each of the two cue types. The final set of individual cue stimuli and estimations of their mean difficulty based on the pilot experiment consisted of the following letters (task demand high: $\mathrm{V}, \mathrm{N}, \mathrm{D}, \mathrm{F}$; low: T, B, S, K) and categories (task demand high: liquids [German: "Flüssigkeiten"], toys ["Spielzeuge"], Furniture ["Möbelstücke"], vegetables ["Gemüsearten"]; low: vehicles/means of transport ["Fortbewegungsmittel"], quadrupeds ["Vierbeiner"], musical instruments ["Musikinstrumente"], professions ["Berufe"]). More detailed information on the behavioral pilot study and stimulus selection can be obtained from the corresponding authors.

Experimental paradigm and design ( $f M R I)$. In the present study, neural correlates of verbal fluency in the LIFG were investigated using a $2 \times 2$ experimental design with factors cue type (phonological vs semantic) and task demand (high vs low). In total, 16 different cue stimuli were applied that were equally allocated to the four cells of the design. Cues were presented visually at the center of a screen that was projected into the scanner bore (see below). After the presentation of a cue stimulus, subjects were prompted 8 times at an interval of $2 \mathrm{~s}$ by an acoustic signal to overtly generate a noun or category member according to the given cue. Cues were presented continuously throughout trials. Verbal fluency trials were interleaved by rest intervals of $8 \mathrm{~s}$. As in the pilot study, subjects were instructed not to use proper names, morphological variations, or repetitions of words previously retrieved in the same trial.

The fMRI experiment comprised three runs, each consisting of 16 trials. A single run lasted for $7.2 \mathrm{~min}$. Individual cue stimuli were repeatedly presented across runs. To avoid potential carry-over effects and to ensure orthogonality of regressors for fMRI modeling, the order of presentation of high- and low-demanding letter and category trials (as well as of individual cues) was pseudorandomly counterbalanced across runs and subjects.

Imaging procedure. $\mathrm{fMRI}$ was performed on a 3.0 tesla whole-body MR Scanner (TIM Trio; Siemens) using an 8-channel head coil. Functional images were collected using 28 oblique axial slices (slice thickness, $4 \mathrm{~mm}$; gap, $1 \mathrm{~mm}$; matrix size, $64 \times 64$; in plane resolution, $3 \times 3 \mathrm{~mm}$; field of view [FOV], $192 \mathrm{~mm}$ ) with a T2*-weighted echoplanar imaging sequence (EPI; repetition time [TR], $1500 \mathrm{~ms}$; echo time [TE], $30 \mathrm{~ms}$; flip angle $[\mathrm{FA}], 70^{\circ}$ ). The experiment comprised a single session with 3 runs each lasting for $7.2 \mathrm{~min} ; 288$ functional images per run and subject were acquired. In-house algorithms were applied for online correction of motion and distortion artifacts (Zaitsev et al., 2004) so that functional im- 
ages were unwarped and spatially aligned already before processing in SPM8 (see below). High-resolution 3D T1-weighted images were obtained with magnetization-prepared rapid gradient echo images (MPRAGE; TR, $2200 \mathrm{~ms}$; TE, $4.11 \mathrm{~ms}$; FA 12² 160 sagittal slices; matrix size, $256 \times 256$; FOV, $256 \mathrm{~mm} ; 1 \mathrm{~mm}^{3}$ cubic voxels).

Subjects lay supine on the scanner bed. To prevent excessive head motion, neck and side pillows were used to fixate subject's head inside the coil. Visual cues were projected onto a screen mounted on the rear of the scanner bore and were viewed via a mirror system. Overt verbal responses were recorded with a combined MR-compatible headset and microphone system (CONFON system; MR confon) that was also used for presentation of an auditory stimulus for pacing responses during verbal fluency trials (see above). A microphone with two orthogonally arranged heads was used to allow for later offline cancellation of scanner noise. Trigger pulses from the MR scanner were monitored by the sound device for subsequent synchronization of verbal responses with functional imaging.

Behavioral data analyses. Recorded audio data were preprocessed and denoised using Audacity (Version 1.3.12; audacity.sourceforge.net). Onsets of verbal responses were automatically detected using Adobe Audition (Version 3; Adobe Systems) and CueListTool (Version 1.7; http://tonbandstimmen.de/cuelisttool/index_e.htm). Subsequent processing was conducted using a custom-built software tool written in MATLAB (MathWorks). At first, onset detection was checked manually and corrected wherever necessary. In addition, verbal responses were listened to and transcribed. Based on trigger pulses synchronously recorded in both audio data and log files from visual presentation, audio data were then merged with information on associated experimental conditions that thus allowed evaluating transcribed verbal responses concerning their accuracy. Consistent with the instructions (see above), any occurrences of proper names, morphological variations, or repetitions of words previously retrieved in the same trial were classified as incorrect responses. Finally, evaluated data were exported for statistical analyses.

Imaging analyses. All preprocessing of imaging data and subsequent statistical analyses were performed using SPM8 (r4667; http://www.fil. ion.ucl.ac.uk/spm/software/spm8). Functional images were coregistered to subjects' anatomical scans. Anatomical scans were segmented using the VBM8 toolbox (r435; http://dbm.neuro.uni-jena.de/software/). Deformation field parameters for nonlinear normalization into the stereotactic Montreal Neurological Institute (MNI) space were then computed using the DARTEL (diffeomorphic anatomical registration through exponentiated lie algebra; Ashburner, 2007) approach implemented in VBM8.

First-level estimates of hemodynamic activation changes were computed based on the General Linear Model. Regressors for each of the four conditions of the $2 \times 2$ factorial design were built by convolving stick functions of presentation onsets with a canonical hemodynamic response function. In addition, the first derivatives of head-motion parameters were entered as nuisance regressors. As first-level analyses were conducted in individual space, resulting $\beta$-images were aggregated across the three sessions and subsequently transformed into stereotactic MNI space using DARTEL deformation fields. Images were resampled to a spatial resolution of $1.5 \times 1.5 \times 1.5 \mathrm{~mm}^{3}$ and smoothed with an isotropic Gaussian kernel with a full width at half maximum of $8 \mathrm{~mm}$.

Normalized $\beta$-images were then entered into second-level analyses of variance based on a flexible factorial model accounting for effects of subjects as well as of the two main effects of interest (cue type, task demand) and their interaction. Because the present research was focused on LIFG, namely BA 44 and BA 45, second-level analyses were masked by cytoarchitectonic probability maps for these areas (Amunts et al., 1999). Probability maps were derived from the SPM Anatomy Toolbox (Version 1.8; Eickhoff et al., 2005, 2006, 2007). Included voxels had a probability of at least $40 \%$ for belonging either to BA 44 or BA 45 (Eickhoff et al., 2005). Significance of activations was assessed at $p<0.05$ (corrected for family-wise error) and a cluster extent of $k>5$ voxels $\left(16.9 \mathrm{~mm}^{3}\right)$.
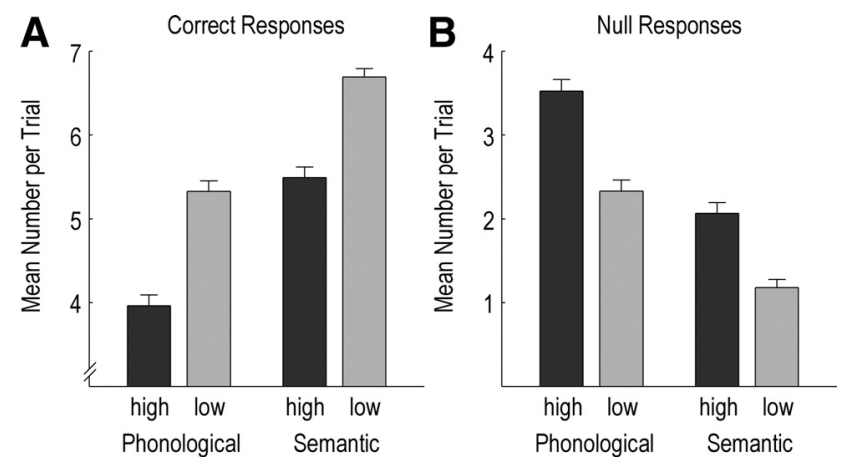

Figure 1. $\quad \boldsymbol{A}, \boldsymbol{B}$, Effects of cue type (lexical vs semantic) and task demand (high vs low) on verbal fluency in terms of the mean number of correct $(\boldsymbol{A})$ and null $(\boldsymbol{B})$ responses. Bars colored in dark and light gray illustrate high and low task demands, respectively. Because the total number of prompted responses was fixed (eight per trial at maximum) and because error rates were very low (4.49\% on average), the observed task-dependent pattern in correct responses was almost perfectly complemented by null responses. Error bars denote SEM.

\section{Results}

\section{Behavioral results}

The mean numbers of correct responses per trial were entered into a repeated-measurements ANOVA with within-subject factors cue type (phonological vs semantic) and task demand (high vs low). As illustrated in Figure $1 A$, results revealed significant main effects of both cue type $\left(F_{(1,61)}=232.99, p<0.001\right.$, partial $\left.\eta^{2}=0.793\right)$ and task demand $\left(F_{(1,61)}=424.34, p<0.001\right.$, partial $\left.\eta^{2}=0.874\right)$, but no significant interaction $\left(F_{(1,61)}=1.38, p=\right.$ 0.245 , partial $\eta^{2}=0.022$ ).

Performance differences between conditions were mainly due to omissions, whereas errors in terms of perseverations, rule breaks, or naming of inadequate class/category members occurred very infrequently. The mean error rate did not exceed $4.49 \%$ of responses. Given that the cued number of responses was fixed to eight responses per trial, observed null responses were therefore almost complementary to the correct responses and not subject to further statistical testing (Fig. 1B).

Because global differences in item difficulty between lexical and semantic cues are a task-inherent and inevitable confound, careful item selection based on prior pilot testing intended to provide comparable levels of difficulty at least for items following phonological and semantic cues with low and high task demands, respectively (see Materials and Methods). A manipulation check in terms of a paired $t$ test confirmed that difficult semantic cues were not significantly more or less demanding than easy phonological cues $\left(t_{(61)}=1.379, p=0.173\right)$. Subsequent calculation of effect size (Cohen's $d=0.1667$ ) further substantiated that this difference did not even constitute a small effect, because this would have required an effect size of $d>0.2$ (Cohen, 1988). Therefore, regardless of a slight difference of 5.33 versus 5.49 responses on average, the two observed distributions for difficult semantic and easy phonological items shared an overlap of $>86 \%$ (Cohen, 1988).

\section{Imaging results}

Second-level analyses comprising a flexible-factorial ANOVA revealed significant main effects of the two within-subject factors cue type (phonological vs semantic) and task demand (high vs low) and a significant interaction. Spatial distributions of activations are illustrated in Figure $2 A, C$. For an allocation of activation patterns to BA 44 and BA 45, results were superimposed on probabilistic cytoarchitectonic maps derived from the SPM 

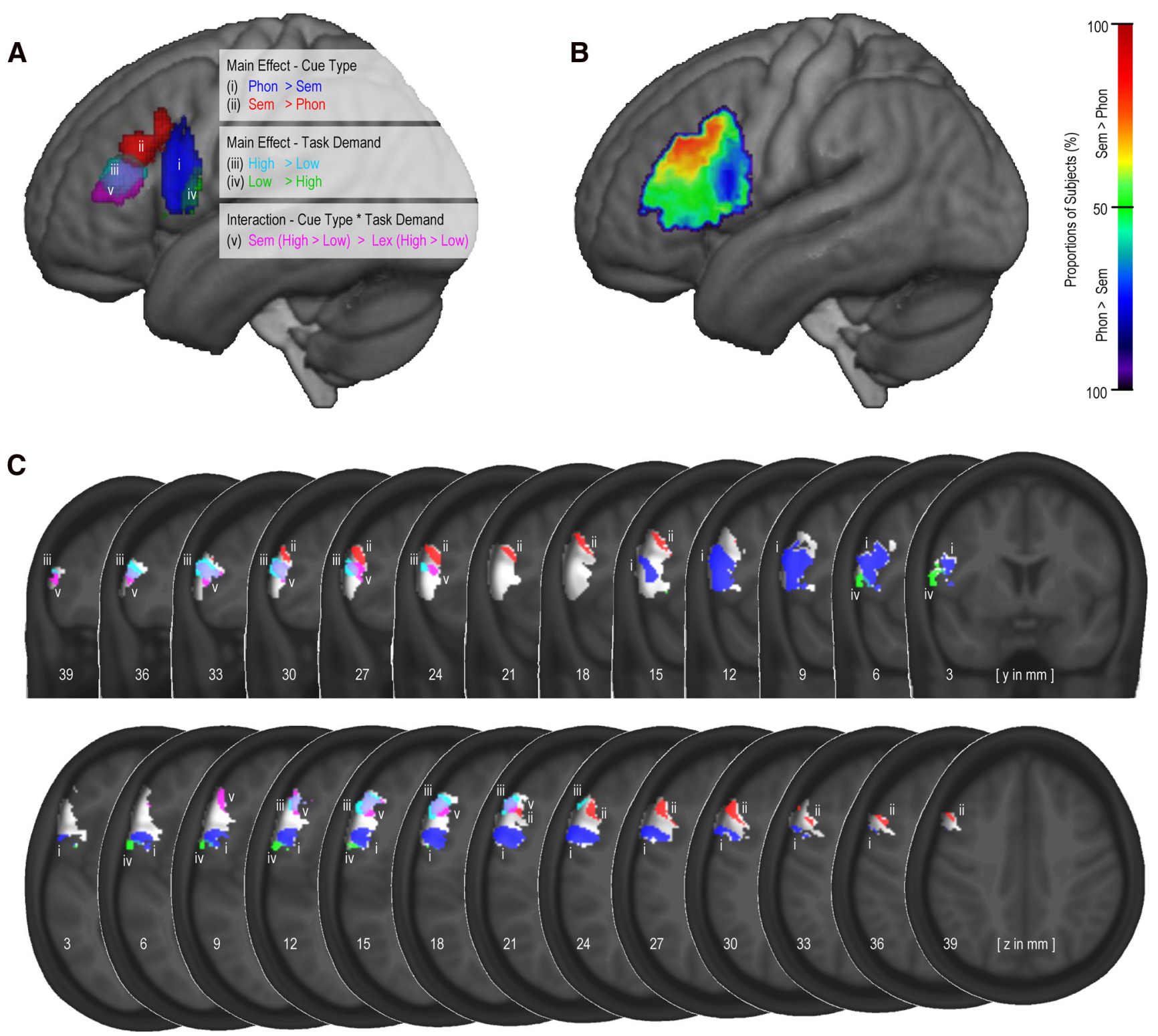

D

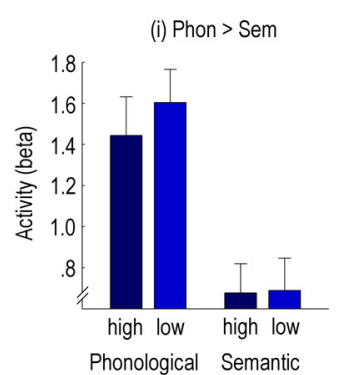

E

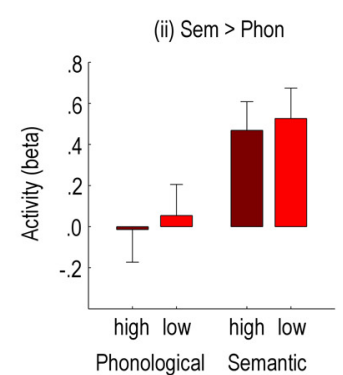

$\mathbf{F}$

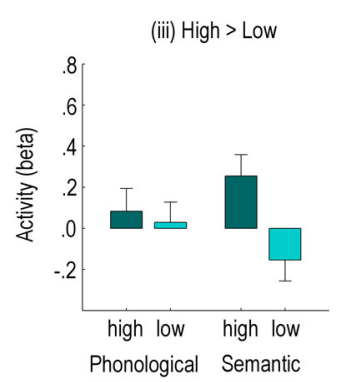

G

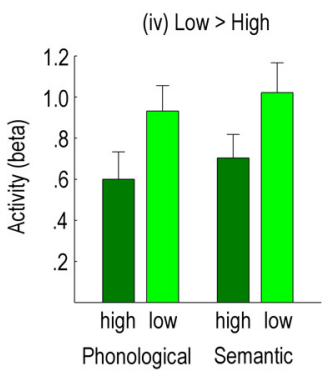

H

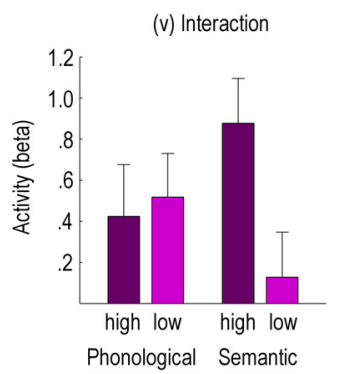

Figure 2. Differential effects of cue type (phonological vs semantic) and task demand (high vs low) on the distribution of activations patterns in LIFG ( $p<0.05$, family-wise error corrected). Colors in $\boldsymbol{A}$ and $\boldsymbol{C} \boldsymbol{H}$ refer to different directions of effects (compare color column in Table 1): (i) Phon $>$ Sem (blue), (ii) Sem $>$ Phon (red), (iii) High $>$ Low (cyan), (iv) Low $>$ High (green), and the interaction (v) Sem (High $>$ Low) $>$ Phon (High $>$ Low) (magenta). A, Rendering of the activation patterns on the average of the sample's normalized brains. $\boldsymbol{B}$, Spatial distribution of the proportions of subjects that showed either a higher activation in semantic versus phonological fluency or vice versa for a given voxel. $C$, Coronar and axial slices illustrating the distribution of activations within BA 44 and BA 45. Activations are projected onto the sample's average template brain in MNI space. Cytoarchitectural mapping of BA 44 and 45 is indicated in light gray (based on probability maps derived from the SPM Anatomy Toolbox, see Materials and Methods), whereas all other parts of the brain are shaded in dark gray. Note that this mapping of BA 44 and BA 45 is identical with the explicit masks used for the analysis. Latin numbers within brain slices indicate spatial locations of main effects and the interaction (see above). Note that effects may spatially overlap, which is indicated by color mixtures (e.g., lilac resulting from the overlap of magenta and cyan). D-H, Parameter estimates for main activation peaks. Means and SEs are calculated based on unadjusted $\beta$ values (thus including random effects of subjects that are statistically accounted for in repeated-measures ANOVA). 
Table 1. Effects of cue type (phonological vs semantic) and task demand (high vs low) on left inferior frontal activation

\begin{tabular}{|c|c|c|c|c|c|c|c|c|c|c|}
\hline \multirow[b]{2}{*}{ Effect } & \multirow[b]{2}{*}{ Color } & \multirow[b]{2}{*}{ Extent } & \multirow[b]{2}{*}{ Area } & \multicolumn{3}{|c|}{ Coordinates } & \multicolumn{4}{|c|}{ Inference statistics } \\
\hline & & & & $x$ & $y$ & $z$ & $t$ & $p_{\mathrm{FWE}}$ & $p_{\text {unc }}$ & $z$ \\
\hline \multicolumn{11}{|l|}{ Cue type } \\
\hline (i) Phon $>$ Sem & & & & -52.5 & 6.0 & 28.5 & 7.14 & $<0.001$ & $<0.001$ & 6.70 \\
\hline \multirow[t]{2}{*}{ (ii) Sem $>$ Phon } & Red & 294 & $45(66.4 \%)$ & -46.5 & 25.5 & 28.5 & 7.65 & $<0.001$ & $<0.001$ & 7.12 \\
\hline & & & & -43.5 & 15.0 & 36.0 & 6.64 & $<0.001$ & $<0.001$ & 6.28 \\
\hline \multicolumn{11}{|l|}{ Task demand } \\
\hline (iv) Low > High & & & & -58.5 & 7.5 & 4.5 & 4.41 & 0.004 & $<0.001$ & 4.29 \\
\hline \multicolumn{11}{|l|}{ Interaction effect } \\
\hline (v) Sem(High > Low) & Magenta & 426 & $45(93.3 \%)$ & -49.5 & 28.5 & 16.5 & 5.84 & $<0.001$ & $<0.001$ & 5.58 \\
\hline$>$ Phon(High $>$ Low) & & & & -43.5 & 33.0 & 12.0 & 5.38 & $<0.001$ & $<0.001$ & 5.17 \\
\hline
\end{tabular}

Color refers to illustration of activation distributions in Figure 2; extent refers to cluster size in voxels $\left(1.5 \times 1.5 \times 1.5 \mathrm{~mm}^{3}\right)$; area denotes the probabilistic cytoarchitectonic mapping of activations with respect to BA 44 and BA 45 ; numbers in parentheses refer to the proportion of the cluster within a given area.

FWE indicates family-wise error correction for multiple comparisons; unc, uncorrected for multiple comparisons.

Anatomy Toolbox (see Materials and Methods; Eickhoff et al., $2005,2006,2007)$. Subsequent comparisons confirmed that, regardless of the level of task demand, phonological cues entailed stronger activations in BA 44 than semantic cues (Fig. 2A, C, activation cluster in blue color), whereas the opposite pattern was observed in posterior dorsal portions of BA 45 (red). In addition, higher compared with lower task demands led to activation differences in anterior dorsal parts of BA 45 (cyan). In contrast, lower compared with higher task demands yielded only an activation difference in posterior parts of BA 44 at the transition to BA 6 (green). Furthermore, activation differences in anterior dorsal portions of BA 45 were driven by a significant interaction in terms of a stronger effect of task demand (high minus low) for semantic cues compared with phonological cues (magenta). Inference statistics are reported in Table 1. Patterns of parameter estimates underlying significant main and interaction effects are illustrated in Figure 2D-H.

So far, present evidence supports the assumption that the differential role of BA 44 and BA 45 for verbal fluency crucially hinges on the combination of two factors: the type of cue and the imposed task demands. However, to corroborate the dissociation proposed by Costafreda et al. (2006) suggesting that stronger contributions of BA 45 under semantic than phonological cues and the opposite pattern for BA 44, an additional flexiblefactorial ANOVA was conducted while controlling for a potential bias due to the global differences in task difficulty between phonological and semantic cues. Therefore, we compared recruitments of BA 44 and BA 45 for phonological versus semantic cues under conditions of similar behavioral performances; that is, hemodynamic responses following phonological cues with low task demands versus semantic cues with high task demands. Results again revealed a significant activation difference $(p<0.05$, family-wise error corrected) for comparing phonological with semantic fluency in BA 44 (peak: - 51.06 .09 .0 ), whereas the opposite pattern was observed in anterior $(-51.036 .016 .5)$ and posterior dorsal parts of BA 45 ( $-48.027 .031 .5)$, although this was less pronounced than in the overall analysis.

Contrasting phonological and semantic fluency revealed spatially and cytoarchitectonically dissociable activation patterns in LIFG. Activation was confined to BA 44 in the frontal operculum for phonological versus semantic fluency and to dorsal portions of BA 45 in the proximity of the inferior frontal sulcus for the reverse comparison. This dissociation also becomes obvious when considering the spatial distribution within BA 44 and BA 45 for the voxelwise proportions of subjects showing an activation difference between phonological and semantic fluency in one or the other direction (Fig. 2B).

\section{Additional post hoc analyses}

As hypothesized, the present analyses revealed that task demand plays a crucial role for detecting activation differences between phonological and semantic fluency in BA 45, particularly in its anterior dorsal portion (Fig. 2G). Most surprisingly, however, results also yielded a main effect for semantic compared with phonological fluency in the posterior dorsal part of BA 45 (Fig. $2 E$ ). Because Heim et al. (2008) did not find any evidence for this dissociation, the resulting discrepancy remains to be clarified. Because the present sample was collected as matched controls for ongoing patient studies, intellectual capabilities of subjects spanned a broader range than the usual student samples commonly recruited for participation in neuroimaging experiments. Therefore, individual task performance as a moderating factor for activation differences might offer a potential explanation for the diverging results on a significant activation difference in LIFG for semantic compared with phonological fluency (in contrast to the findings of Heim et al., 2008). To test this assumption, subjects were split into two subsamples based on the empirical estimates of their average performance scores in verbal fluency above/below the overall sample's median (across all 4 conditions of the $2 \times 2$ experimental design, i.e., phonological vs semantic cues by high vs low task demands). Resulting subsamples did not differ with respect to age (high performer, $26.61 \pm 7.75$ years; low performer, $25.56 \pm 5.33$ years; $\left.t_{(60)}=0.62, p=0.538\right)$; male and female subjects were equally distributed $\left(\chi_{(1)}=0.261, p=0.610\right)$ across subsamples (high performer, 18 males; low performer, 16 males). For 52 of the 62 subjects ( 24 low vs 28 high performer), an estimation of verbal crystallized intelligence based on a German vocabulary test was available (the Mehrfachwahl-WortschatzTest; Lehrl, 1999). As expected (Borkowski et al., 1967), subjects with a high performance in verbal fluency also had significantly higher intelligence scores than low-performing subjects $\left(t_{(50)}=\right.$ 2.53, $p=0.014$, Cohen's $d=0.72$ ).

With respect to the mean number of correct responses per trial, a repeated-measures ANOVA including subsample as an additional between-subjects factor further revealed a main effect of subsample $\left(F_{(1,61)}=96.83, p<0.001\right)$, but also a marginally significant interaction between cue type and subsample $\left(F_{(1,60)}=\right.$ $3.83, p=0.055)$. No other two- or three-way interaction with subsample approached significance (highest $F=0.55$, lowest $p=$ 


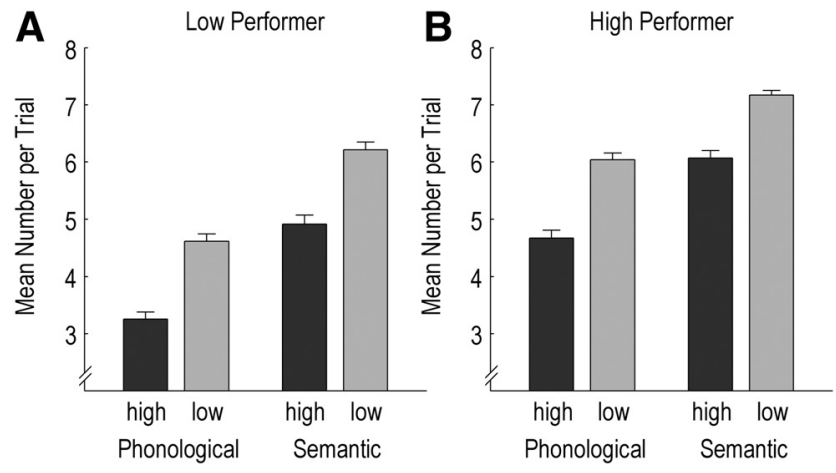

Figure 3. Effects of cue type (lexical vs semantic) and task demand (high vs low) on the mean number of correct responses in low-performing $(\boldsymbol{A})$ and high-performing subjects $(\boldsymbol{B})$. Error bars denote SEM.
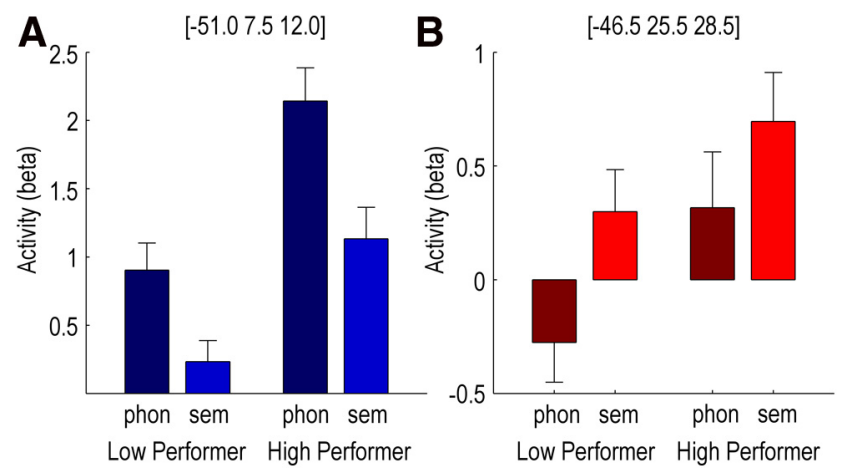

Figure 4. Influence of subjects' ability on left inferior frontal activation differences imposed by cue type in BA $44(\boldsymbol{A})$ and posterior dorsal BA $45(\boldsymbol{B})$. Assignment of bar colors is identical to Figure $2 A$ and refers to the different directions of effects in the main analysis: Phon $>$ Sem (blue), Sem $>$ Phon (red). Means and SEs are calculated based on unadjusted $\beta$ values (thus including random effects of subjects that are accounted for in repeated-measures ANOVA).

0.462). Behavioral performance of both subsamples is illustrated in Figure $3 A, B$.

To explore the influence of individual ability and task performance on the activation patterns reported in the main analysis (Fig. 1), additional analyses were computed based on individual $\beta$ values extracted at group coordinates of the five major peak voxels (Table 1). Given that the primary contrasts for identification of seeds for extraction were independent of subsequent statistical tests on the effects of interest concerning the factor sample (high vs low performer) and its interactions, biases in terms of double dipping were effectively prevented (Kriegeskorte et al., 2009). Results revealed a significant interaction effect between cue type and sample in BA 44 (Fig. 1, activation cluster in red; $\left.F_{(1,60)}=5.22, p=0.026\right)$ and a trend in posterior dorsal BA 45 (Fig. 1, activation cluster in blue; $F_{(1,60)}=3.02, p=0.087$ ), but not in the other LIFG peaks (green, magenta, cyan; highest $F=$ 0.331 , lowest $p=0.567$ ). As illustrated in Figure $4 A$, stronger BA 44 activations in conditions of phonological compared with semantic fluency were evident particularly in subjects with higher task performance, whereas the opposite contrast was more pronounced in posterior dorsal BA 45 in low-performing subjects (Fig. 4B). Summarizing the additional analyses, individual ability seems to be another determinant for dissociable recruitments of LIFG in phonological and semantic fluency.

\section{Discussion}

Cross-study comparisons in a recent meta-analysis suggested that semantic and phonological fluency are subserved to different
Table 2. Approximation of item difficulty in the semantic fluency condition of Heim et al. (2008) based on Mannhaupt (1983)

\begin{tabular}{llll}
\hline \multirow{2}{*}{$\begin{array}{l}\text { Heim et al. } \\
\text { Item }\end{array}$} & Mannhaupt & & \\
\cline { 2 - 3 } & Related item (\#) & Search field & Expected difficulty \\
\hline Birds & "Vogel" (36) & 15.65 & Low \\
Mammals & NA & NA & NA \\
Food & "Nahrungsmittel" (21) & 13.87 & Low \\
Weapons & NA & NA & NA \\
Tools & "Werkzeug" (31) & 8.69 & High \\
Toys & "Spielzeug" (14) & 9.65 & High \\
\hline
\end{tabular}

Because Heim et al. (2008) tested German natives, item difficulty of the applied semantic categories can be approximated using normative data from a German sample reported by Mannhaupt (1983). The variable "search field" is an empirically derived estimation of the size of a given semantic category that reflects its difficulty; a larger search field is associated with a lower difficulty and vice versa. NA indicates not assessed.

extents by anterior-ventral and posterior-dorsal parts of LIFG, respectively (Costafreda et al., 2006). Heim et al. (2008) attempted to validate this proposal in a within-subjects experiment, but did not find evidence for the suggested dissociation. The present results resolve this apparent discrepancy. By taking into account differences in task demands and individual ability, we found a functional segregation of LIFG that accommodates both the proposal of Costafreda et al. (2006) and the results of Heim et al. (2008). Therefore, neural activation observed in BA 44 and BA 45 is differentially associated with information processing involved in phonological and semantic search. However, stronger activation in semantic fluency in anterior and posterior parts of dorsal BA 45 appeared to be differentially driven by cue difficulty and subjects' performance (Figs. 2, 4).

These present findings have several implications. First of all, they provide a compelling explanation as to why Heim et al. (2008) and others (Mummery et al., 1996; Gourovitch et al., 2000) failed to find stronger involvement of BA 45 in semantic compared with phonological fluency. The main effect of semantic versus phonological fluency was shown to be performance dependent and particularly evident in low-performing subjects (Fig. 4B). Moreover, because Heim et al.'s set of semantic cues incorporated both difficult and easy items (Table 2), any specific contributions of BA 45 under semantic fluency conditions with high task demands would most likely be attenuated.

The evidence presented in this study for a differential involvement of BA 45 in semantic fluency compared with phonological fluency may also help to resolve controversial findings in the extant lesion literature on whether LIFG is specifically involved in semantic fluency. Although phonological fluency is particularly sensitive to left frontal damage (Milner, 1964; Benton, 1968; Stuss et al., 1998; Robinson et al., 2012), findings of impaired performance for semantic fluency are less unequivocal (for an overview, see Troyer et al., 1998, but also see meta-analysis of Henry and Crawford, 2004). Using a voxel-based lesion-symptom mapping approach, Baldo et al. (2006) suggested that the degree of frontal involvement may rely on differences in the task demands of semantic categories. This has now been corroborated by the present data in that activation of anterior-dorsal BA 45 (Fig. 2, magenta) was driven by an interaction effect between task demand and cue type. Therefore, task-demand-related activation differences were most pronounced in semantic but not phonological fluency, presumably indicating different demands on cognitive control.

Previous research has proposed a nonlinguistic role of LIFG subregions in being responsible for domain-independent selection from among competing alternatives (Thompson-Schill et al., 1997, 1998; Robinson et al., 1998). In verbal fluency, a semantically cued search is predicated primarily on spreading activa- 
tions in semantic networks, thus requiring a controlled selection from among coactivated items. Higher demands on controlled item selection can therefore be expected for more extensive, less restrained semantic categories that activate a multitude of alternative response options. Here, however, the easier semantic condition with low task demands was associated with the least activation in anterior-dorsal BA 45 (Fig. $2 \mathrm{H}$, magenta), thus rendering this interpretation unlikely. Therefore, differential demands on the selection of appropriate semantic representations can only partially account for the present data in so far as the more difficult of the semantic conditions requires to restrain inadequate and distracting items from adjacent but irrelevant categories (Troyer et al., 1997, Unsworth et al., 2011) or to suppress recurrent resampling of previously retrieved items (Rosen and Engle, 1997). Phonological fluency may place even higher demands on selection because it requires suppression of habitual responses automatically triggered by the meaning of retrieved words (Perret, 1974, but also see Henry and Crawford, 2004; Robinson et al., 2012).

LIFG's proposed pivotal role in controlled semantic retrieval (Wagner et al., 2001) may provide an alternative interpretation of the present interaction of cue type and task demands in anteriordorsal BA 45 (Fig. 2, magenta). LIFG is suggested to "directly support the top-down (controlled) retrieval of knowledge when bottom-up (automatic) retrieval is insufficient" (Badre et al., 2005, p. 908; see also Wagner et al., 2001). In semantic fluency, searching for members of a given category (e.g., fruits) is believed to be a two-stage process (Gruenewald and Lockhead, 1980), consisting of a top-down identification of task-relevant subcategories in terms of semantically related search fields (e.g., citrus fruits) and a bottom-up retrieval of items based on automatically triggered associations (i.e., spreading activations; Collins and Loftus, 1975) within that subcategory (e.g., orange-tangerinelemon-etc.; Reverberi et al., 2006). Therefore, when a subcategory (e.g., citrus fruits) is exhausted so that automatic coactivation of new items based on semantic proximity is weak, another task-relevant subcategory (e.g., berries) has to be actively identified and switched to (Gruenewald and Lockhead, 1980; Rosen and Engle, 1997; Troyer et al., 1997, 1998; Mayr and Kliegl, 2000). Accordingly, semantic categories with small search fields are more difficult (compare Table 2, Mannhaupt, 1983) because they place increased demands on strategic search and generation of new subcategories. Consistent with this, anterior-dorsal BA 45 was most strongly activated in semantic fluency with high task demands and least engaged in semantic fluency with low task demands (Fig. $2 \mathrm{H}$, magenta). In contrast, anterior-dorsal BA 45 showed an intermediate activation in both phonological conditions (Paulesu et al., 1997); that is, regardless of task demands. This result is consistent with assumptions that phonological search is not exclusively based on phonemic information, but may also rely on semantic facilitation (Schwartz et al., 2003), possibly reflecting deliberate application of semantic retrieval strategies serving to structure the search in phonological fluency (Azuma, 2004). For example, searching words of the given initial letter (e.g., T) may be guided by semantic categories (animals starting with $\mathrm{T}$, then fruits, etc.).

Concurring with the proposed LIFG mechanisms on topdown control in semantic retrieval, patients with lesions in lateral frontal cortex were found to be specifically impaired in strategic search organization, manifested by an increased number of switches between subcategories and therefore a reduced semantic relatedness between subsequently retrieved items, whereas the overall number of produced subcategories was unaffected (Reverberi et al., 2006). It was further proposed that an efficient strategy particularly affects fluency for smaller semantic categories (Baldo et al., 2006; Reverberi et al., 2006), which is corroborated by the present interaction in anterior-dorsal BA 45 (Fig. $2 H$ ). Inconsistent findings on the sensitivity of semantic fluency for frontal lobe damage (Troyer et al., 1998) may thus be caused by use of large semantic categories that allow for an automatically triggered collection of items based on spreading activations, thus exerting only low demands on top-down guided retrieval. Furthermore, given that the present results suggest that controlled semantic retrieval is localized in anterior-dorsal BA 45, distributions of lesion in previous studies may have affected posterior parts of LIFG relevant for phonological fluency to a greater extent (compare Stuss et al., 1998, Robinson et al., 2012) than anterior parts of LIFG related to semantic fluency.

Finally, the present data demonstrate that domain-specific activation differences exist in both directions (Fig. 2), but are restricted to posterior-dorsal BA 45 (semantic > phonological; red) and ventral BA 44 (phonological > semantic; blue). These opposing effects may be associated with different cognitive processes preceding the generation of correct responses in the two types of verbal fluency. Although not directly demonstrated for verbal fluency tasks until now, an anterior-posterior gradient in LIFG for semantic versus phonological processing was related to BA 44 and BA 45 previously (Poldrack et al., 1999; McDermott et al., 2003). However, against earlier claims for a domain-specific parcellation of LIFG, the differential activation, particularly in BA 45, most likely originates from unequal demands on cognitive control imposed by phonological and semantic tasks (Gold and Buckner, 2002; Gold et al., 2005).

The present evidence for a functional segregation of BA 45 is also consistent with anatomical findings derived from multiple-receptor mapping in humans (Amunts et al., 2010) and cytoarchitectonic parcellations in macaque monkeys (Petrides and Pandya, 1994, 2009; Gerbella et al., 2007), both of which suggested a subdivision of BA 45 into an anterior part (45A) and a posterior part (45B). In primates, the existence of two distinct subareas within BA 45 is further supported by divergent anatomical connectivity of BA 45A and 45B with other cortical (Gerbella et al., 2010) and subcortical areas (Contini et al., 2010). Previous neuroimaging experiments on language-related processing revealed a functional segregation of ventral and dorsal BA 44 (Makuuchi et al., 2009; see also Friederici, 2011). The present data suggest a similar parcellation of BA 45 into several functionally (and most likely also anatomically) distinct subdivisions. However, relating the present results to cytoarchitecture also warrants some caution, because the applied probability maps may overestimate the spatial extent of BA 44 and BA 45 toward unmapped cortex in dorsally adjacent BA 9 and BA 9/46 (Eickhoff et al., 2005). That is, the dissociation pattern in Figure $2 B$ may partially reflect functions of middle frontal gyrus, into which the present activations extend.

Taken together, the present findings considerably expand previous accounts on the role of LIFG in verbal fluency by providing explanations for inconsistent findings in both the extant neuroimaging and neuropsychological literature. Phonological fluency is most likely triggered by subvocal syllabification (Rende et al., 2002) that overlaps with processes of inner speech such as motor programming and articulation (Indefrey and Levelt, 1999), as indicated by stronger activations of posterior LIFG (BA 44; Fig. 2, blue) close to adjacent 
(pre)motor areas. In contrast, search in semantic fluency seems to rely particularly on top-down mechanisms of cognitive control that are, however, task dependent and only critical if automatic retrieval is insufficient. Therefore, one would expect lesions of LIFG to result in marked deficits of phonological fluency, whereas semantic fluency should be affected only under high task demands on controlled retrieval.

\section{References}

Amunts K, Schleicher A, Bürgel U, Mohlberg H, Uylings HB, Zilles K (1999) Broca's region revisited: cytoarchitecture and intersubject variability. J Comp Neurol 412:319-341. CrossRef Medline

Amunts K, Weiss PH, Mohlberg H, Pieperhoff P, Eickhoff S, Gurd JM, Marshall JC, Shah NJ, Fink GR, Zilles K (2004) Analysis of neural mechanisms underlying verbal fluency in cytoarchitectonically defined stereotaxic space-the roles of Brodmann areas 44 and 45 . Neuroimage 22:42-56. CrossRef Medline

Amunts K, Lenzen M, Friederici AD, Schleicher A, Morosan P, PalomeroGallagher N, Zilles K (2010) Broca's region: novel organizational principles and multiple receptor mapping. PLoS Biol 8:e1000489.

Ashburner J (2007) A fast diffeomorphic image registration algorithm. Neuroimage 38:95-113. CrossRef Medline

Azuma T (2004) Working memory and perseveration in verbal fluency. Neuropsychology 18:69-77. CrossRef Medline

Badre D, Poldrack RA, Paré-Blagoev EJ, Insler RZ, Wagner AD (2005) Dissociable controlled retrieval and generalized selection mechanisms in ventrolateral prefrontal cortex. Neuron 47:907-918. CrossRef Medline

Baldo JV, Schwartz S, Wilkins D, Dronkers NF (2006) Role of frontal versus temporal cortex in verbal fluency as revealed by voxel-based lesion symptom mapping. J Int Neuropsychol Soc 12:896-900. CrossRef Medline

Benton AL (1968) Differential behavioral effects in frontal lobe disease. Neuropsychologia 6:53-60. CrossRef Medline

Birn RM, Kenworthy L, Case L, Caravella R, Jones TB, Bandettini PA, Martin A (2010) Neural systems supporting lexical search guided by letter and semantic category cues: a self-paced overt response fMRI study of verbal fluency. Neuroimage 49:1099-1107. CrossRef Medline

Borkowski JG, Benton AL, Spreen O (1967) Word fluency and brain damage. Neuropsychologia 5:135-140. CrossRef

Cohen J (1988) Statistical power analysis for the behavioral sciences ( $2^{\text {nd }}$ ed). Mahwah, NJ: Lawrence Erlbaum Associates.

Collins AM, Loftus EF (1975) A spreading-activation theory of semantic processing. Psych Rev 82:407-428. CrossRef

Contini M, Baccarini M, Borra E, Gerbella M, Rozzi S, Luppino G (2010) Thalamic projections to the macaque caudal ventrolateral prefrontal areas 45A and 45B. Eur J Neurosci 32:1337-1353. CrossRef Medline

Costafreda SG, Fu CH, Lee L, Everitt B, Brammer MJ, David AS (2006) A systematic review and quantitative appraisal of fMRI studies of verbal fluency: role of the left inferior frontal gyrus. Hum Brain Mapp 27:799810. CrossRef Medline

Eickhoff SB, Stephan KE, Mohlberg H, Grefkes C, Fink GR, Amunts K, Zilles K (2005) A new SPM toolbox for combining probabilistic cytoarchitectonic maps and functional imaging data. Neuroimage 25:1325-1335. CrossRef Medline

Eickhoff SB, Heim S, Zilles K, Amunts K (2006) Testing anatomically specified hypotheses in functional imaging using cytoarchitectonic maps. Neuroimage 32:570-582. CrossRef Medline

Eickhoff SB, Paus T, Caspers S, Grosbras MH, Evans AC, Zilles K, Amunts K (2007) Assignment of functional activations to probabilistic cytoarchitectonic areas revisited. Neuroimage 36:511-521. CrossRef Medline

Friederici AD (2011) The brain basis of language processing: from structure to function. Physiol Rev 91:1357-1392. CrossRef Medline

Gerbella M, Belmalih A, Borra E, Rozzi S, Luppino G (2007) Multimodal architectonic subdivision of the caudal ventrolateral prefrontal cortex of the macaque monkey. Brain Struct Function 212(3-4):269-301.

Gerbella M, Belmalih A, Borra E, Rozzi S, Luppino G (2010) Cortical connections of the macaque caudal ventrolateral prefrontal areas $45 \mathrm{~A}$ and 45B. Cereb Cortex 20:141-168. CrossRef Medline

Gold BT, Buckner RL (2002) Common prefrontal regions coactivate with dissociable posterior regions during controlled semantic and phonological tasks. Neuron 35:803-812. CrossRef Medline

Gold BT, Balota DA, Kirchhoff BA, Buckner RL (2005) Common and dis- sociable activation patterns associated with controlled semantic and phonological processing: evidence from FMRI adaptation. Cereb Cortex 15: 1438-1450. CrossRef Medline

Gourovitch ML, Kirkby BS, Goldberg TE, Weinberger DR, Gold JM, Esposito G, Van Horn JD, Berman KF (2000) A comparison of rCBF patterns during letter and semantic fluency. Neuropsychology 14:353-360. CrossRef Medline

Gruenewald PJ, Lockhead GR (1980) The free recall of category examples. J Exp Psychol Hum Learn Mem 6:225-240. CrossRef

Heim S, Eickhoff SB, Amunts K (2008) Specialisation in Broca's region for semantic, phonological, and syntactic fluency? Neuroimage 40: 1362-1368. CrossRef Medline

Henry JD, Crawford JR (2004) A meta-analytic review of verbal fluency performance following focal cortical lesions. Neuropsychology 18 : 284-295. CrossRef Medline

Indefrey P, Levelt W (1999) The neural correlates of language production. In M.S. Gazzaniga (ed), The new cognitive neurosciences, Ed 2, pp 845866. Cambridge, MA: MIT.

Kriegeskorte N, Simmons WK, Bellgowan PS, Baker CI (2009) Circular analysis in systems neuroscience: the dangers of double dipping. Nat Neurosci 12:535-540. CrossRef Medline

Lehrl S (1999) Mehrfachwahl-Wortschatz-Intelligenztest MWT-B. Balingen: Spitta Verlag.

Lezak MD, Howieson DB, Loring DW (2004) Neuropsychological assessment. New York: Oxford University.

Makuuchi M, Bahlmann J, Anwander A, Friederici AD (2009) Segregating the core computational faculty of human language from working memory. Proc Natl Acad Sci U S A 106:8362-8367. CrossRef Medline

Mannhaupt HR (1983) Produktionsnormen für verbale Reaktionen zu 40 geläufigen Kategorien. Sprache und Kognition 4:264-278.

Mayr U, Kliegl R (2000) Complex semantic processing in old age: Does it stay or does it go? Psychol Aging 15:29-43. CrossRef Medline

McDermott KB, Petersen SE, Watson JM, Ojemann JG (2003) A procedure for identifying regions preferentially activated by attention to semantic and phonological relations using functional magnetic resonance imaging. Neuropsychologia 41:293-303. CrossRef Medline

Milner B (1964) Some effects of frontal lobectomy in man. In: Warren JM, Akert K (eds), The frontal granular cortex and behavior, pp 313-331. New York: McGraw-Hill.

Mitrushina MN, Boone KB, D’Elia LF (1999) Handbook of normative data for neuropsychological assessment. New York: Oxford University.

Mummery CJ, Patterson K, Hodges JR, Wise RJ (1996) Generating 'tiger' as an animal name or a word beginning with $\mathrm{T}$ : differences in brain activation. Proc Biol Sci 263:989-995. CrossRef Medline

Paulesu E, Goldacre B, Scifo P, Cappa SF, Gilardi MC, Castiglioni I, Perani D, Fazio F (1997) Functional heterogeneity of left inferior frontal cortex as revealed by fMRI. Neuroreport 8:2011-2017. CrossRef Medline

Perret E (1974) The left frontal lobe of man and the suppression of habitual responses in verbal categorical behavior. Neuropsychologia 12:323-330. CrossRef Medline

Petrides M, Pandya DN (1994) Comparative architectonic analysis of the human and the macaque frontal cortex. In: Handbook of neuropsychology (Boller F, Grafman J, eds). Amsterdam: Elsevier.

Petrides M, Pandya DN (2009) Distinct parietal and temporal pathways to the homologues of Broca's area in the monkey. PLoS Biol 7:e1000170. CrossRef Medline

Poldrack RA, Wagner AD, Prull MW, Desmond JE, Glover GH, Gabrieli JD (1999) Functional specialization for semantic and phonological processing in the left inferior prefrontal cortex. Neuroimage 10:15-35. CrossRef Medline

Rende B, Ramsberger G, Miyake A (2002) Commonalities and differences in the working memory components underlying letter and category fluency tasks: a dual-task investigation. Neuropsychology 16:309-321. CrossRef Medline

Reverberi C, Laiacona M, Capitani E (2006) Qualitative features of semantic fluency performance in mesial and lateral frontal patients. Neuropsychologia 44:469-478. CrossRef Medline

Robinson G, Blair J, Cipolotti L (1998) Dynamic aphasia: An inability to select between competing verbal responses? Brain 121:77-89. CrossRef Medline

Robinson G, Shallice T, Bozzali M, Cipolotti L (2012) The differing roles of 
the frontal cortex in fluency tests. Brain 135:2202-2214. CrossRef Medline

Rosen VM, Engle RW (1997) The role of working memory capacity in retrieval. J Exp Psychol Gen 126:211-227. CrossRef Medline

Schwartz S, Baldo J, Graves RE, Brugger P (2003) Pervasive influence of semantics in letter and category fluency: a multidimensional approach. Brain Language 87:400-411. CrossRef Medline

Strauss E, Sherman EMS, Spreen O (2006) A compendium of neuropsychological tests. Oxford, UK: University.

Stuss DT, Alexander MP, Hamer L, Palumbo C, Dempster R, Binns M, Levine B, Izukawa D (1998) The effects of focal anterior and posterior brain lesions on verbal fluency. J Int Neuropsychol Soc 4:265-278. Medline

Thompson-Schill SL, D'Esposito M, Aguirre GK, Farah MJ (1997) Role of left inferior prefrontal cortex in retrieval of semantic knowledge: a reevaluation. Proc Natl Acad Sci U S A 94:14792-14797. CrossRef Medline

Thompson-Schill SL, Swick D, Farah MJ, D’Esposito M, Kan IP, Knight RT (1998) Verb generation in patients with focal frontal lesions: a neuropsychological test of neuroimaging findings. Proc Natl Acad Sci U S A 95: 15855-15860. CrossRef Medline
Troyer AK, Moscovitch M, Winocur G (1997) Clustering and switching as two components of verbal fluency: evidence from younger and older healthy adults. Neuropsychology 11:138-146. CrossRef Medline

Troyer AK, Moscovitch M, Winocur G, Alexander MP, Stuss D (1998) Clustering and switching on verbal fluency: the effects of focal frontal- and temporal-lobe lesions. Neuropsychologia 36:499-504. CrossRef Medline

Unsworth N, Spillers GJ, Brewer GA (2011) Variation in verbal fluency: a latent variable analysis of clustering, switching, and overall performance. Q J Exper Psychol 64:447-466. CrossRef Medline

Wagner AD, Paré-Blagoev EJ, Clark J, Poldrack RA (2001) Recovering meaning: left prefrontal cortex guides controlled semantic retrieval. Neuron 31:329-338. CrossRef Medline

Zaitsev M, Hennig J, Speck O (2004) Point spread function mapping with parallel imaging techniques and high acceleration factors: fast, robust, and flexible method for echo-planar imaging distortion correction. Magn Reson Med 52:1156-1166. CrossRef Medline 